\title{
Report
}

\section{BMI: Gesetzentwurf zur Verbesserung der IT-Sicherheit}

Bundesinnenminister Dr. Friedrich hat am 05.03.2013 einen Gesetzentwurf zur Verbesserung der IT-Sicherheit kritischer Infrastrukturen vorgelegt. Der Entwurf' befindet sich in der Abstimmung zwischen den Ministerien und wurde den betroffenen Verbände zur Stellungnahme zugeleitet.

Zentrale Regelungsinhalte zur Verbesserung der IT-Sicherheit

- Pflicht zur Erfüllung von Mindestanforderungen an IT-Sicherheit für Betreiber kritischer Infrastrukturen: Die Betreiber der wichtigsten kritischen Infrastrukturen sollen IT-Sicherheitsmaßnahmen nach dem Stand der Technik ergreifen und ihre Einhaltung sicherstellen. Branchen können brancheninterne Standards entwickeln, die das Bundesamt für die Sicherheit in der Informationstechnik (BSI) als Konkretisierung der gesetzlichen Verpflichtung anerkennt.

- Pflicht zur Meldung erheblicher IT-Sicherheitsvorfälle für Betreiber kritischer Infrastrukturen: Die Betreiber der wichtigsten kritischen Infrastrukturen sollen dem BSI unverzüglich IT-Sicherheitsvorfälle mit Auswirkungen auf die Versorgungssicherheit oder die öffentliche Sicherheit über hierfür etablierte Wege melden. Nur so ist zu gewährleisten, dass das Bundesamt ein valides nationales Lagebild erstellen und die Betreiber bei Bewältigung des Vorfalls unterstützten kann.

- Pflicht zur Erfüllung von Mindestanforderungen an IT-Sicherheit für Telekommunikationsanbieter: Die Anbieter sollen IT-Sicherheit nach dem Stand der Technik nicht nur wie bisher zum Vertraulichkeitsschutz und zum Schutz personenbezogener Daten, sondern auch zum Schutz vor unerlaubten Eingriffen in die Infrastruktur gewährleisten, um die Widerstandsfähigkeit der Netze insgesamt zu verbessern und damit die Verfügbarkeit zu sichern.

- Pflicht zur Meldung erheblicher IT-Sicherheitsvorfälle für Telekommunikationsanbieter: Die Anbieter sollen IT-Sicherheitsvorfälle, die zu einer Störung der Verfügbarkeit oder zu einem unerlaubte Zugriff auf Systeme der Nutzer führen können, unverzüglich melden. Über die bestehende Meldeverpflichtung im Falle der Verletzung des Schutzes personenbezogener Daten hinaus, wird so gewährleistet, dass die für das Rückgrat der Informationsgesellschaft verantwortlichen Anbieter zu einem validen und vollständigen Lagebild beitragen.

- Verpflichtung der Telekommunikationsanbieter zur Information der Nutzer über Schadprogramme und zur Bereitstellung technischer Hilfsmittel für ihre Erkennung und Beseitigung: Die vorgeschriebene Information soll die Nutzer in die Lage versetzen, selbst Maßnahmen gegen Schadsoftware zu ergreifen. Außerdem sollen die Anbieter den Nutzern einfach bedienbare Sicherheitswerkzeuge bereitstellen, die vorbeugend genutzt werden

1 http://www.bmi.bund.de/SharedDocs/Downloads/DE/Gesetzestexte/ Entwuerfe/Entwurf_it-sicherheitsgesetz.pdf können und auch zur Beseitigung von Störungen, die vom infizierten System des betroffenen Nutzers ausgehen.

- Pflicht zur Erfüllung von Mindestanforderungen an IT-Sicherheit für Telemediendiensteanbieter: Um Verbreitung von Schadprogrammen über Telemedien zu reduzieren, sollen die Anbieter, die Telemediendienste geschäftsmäßig und gegen Entgelt anbieten, verpflichtet werden, anerkannte Schutzmaßnahmen zur Verbesserung der IT-Sicherheit in einem zumutbaren Umfang umzusetzen.

- Jährliche Berichtspflicht des BSI: Durch den vorgesehenen Jahresbericht und dessen Veröffentlichung soll die weitere Sensibilisierung der Bevölkerung für das Thema "IT-Sicherheit" erreicht werden, welche in Anbetracht der Tatsache, dass eine Vielzahl von erfolgreichen IT-Angriffen bei Einsatz von Standardwerkzeugen zu verhindern gewesen wären, von besonderer Bedeutung ist.

\section{Neue Forschungsprojekte zu IT-Sicherheit in eingebetteten Systemen gestartet}

"Internet der Dinge“ und "Industrie 4.0" - diese zwei Begriffe stehen für die rasant fortschreitende Vernetzung unseres Lebens. Die Kommunikation zwischen Geräten und Maschinen - das „Internet der Dinge" - entwickelt sich immer mehr zu einer Basis für das Funktionieren moderner Industriegesellschaften. Vor allem in der industriellen Produktion und der Telemedizin ermöglichen vernetzte intelligente Systeme, die in Maschinen und Geräte eingebettet sind, dass diese autonom miteinander kommunizieren. So werden völlig neue Funktionalitäten und Geschäftsmodelle möglich. Durch die zunehmende Vernetzung steigt aber auch das Risiko, dass diese Netzwerke Ziel von Cyberangriffen werden, die nicht nur erheblichen finanziellen Schaden verursachen, sondern auch die Funktionssicherheit technischer Anlagen bedrohen.

Im Kontext der 4. Ausschreibungsrunde des gemeinsam vom Bundesministerium für Bildung und Forschung und dem Bundesministerium des Innern initiierten Programms "IT-Sicherheitsforschung" sind jetzt drei Forschungsprojekte an den Start gegangen, die neue Lösungen für eingebettete Systeme und eine hohe Sicherheit bei der Übertragung durch neue Verschlüsselungsmethoden zum Ziel haben. Beteiligt sind Hochschulen, Forschungseinrichtungen und Unternehmen.

„Mit dem gemeinsamen Programm IT-Sicherheitsforschung tragen wir dazu bei, das Internet sicherer und zuverlässiger zu machen und den Datenschutz noch weiter zu verbessern. Dies ist eine wichtige Voraussetzung dafür, dass Deutschland seine Spitzenposition als sicherer Wirtschaftsstandort beibehält", sagte die Bundesministerin für Bildung und Forschung, Johanna Wanka, anlässlich des Projektstarts.

Der Bundesminister des Innern, Hans-Peter Friedrich, betonte: „Die Bedrohung von vernetzen IT-Systemen und Produktionsanla- 